\title{
Aplicação de Transformadas Diferenciais na Solução Numérica de Equações Diferenciais Parabólicas
}

\author{
Cirus Caio Nóbrega Barbosa Emerson Alexandre de Oliveira Lima \\ Programa de Pós-Graduação em Engenharia de Sistemas, PPGES-UPE \\ Escola Politécnica da Universidade de Pernambuco \\ Rua Benfica, 455 - Madalena - Recife/PE CEP: 50720-001 \\ E-mail: ciruscaio@gmail.com, eal@poli.br,
}

\section{RESUMO}

Equações diferenciais ordinárias (EDO's) e equações diferenciais parciais (EDP's) são algumas das ferramentas mais fundamentais na modelagem de fenômenos físicos encontrados em todos os ramos da ciência e engenharia [3].

Via-de-regra, equações diferenciais gerais não possuem solução analítica ou esta, quando existe, pode ser de difícil obtenção, desta forma, métodos numéricos de aproximação das soluções têm sido extensivamente utilizados e a pesquisa na área é bastante intensiva. Enquanto que o Teorema da Existência e Unicidade fornece - para o caso de equações diferenciais ordinárias - condições gerais que garantem a estabilidade numérica das soluções aproximadas, no caso das EDPs o problema é bem mais complexo e a própria garantia das condições de contorno mínimas exigidas para existência das soluções é um problema bastante complexo.

Neste trabalho, desenvolvido durante o primeiro ano do mestrado do primeiro autor, descrevemos uma técnica recente - denominada de Transformada Diferencial[1] - para obtenção tanto de soluções exatas quanto de aproximações numéricas da solução de equações diferenciais parciais. A técnica é ilustrada na solução exata e numérica da equação do calor e os resultados são comparados com outras técnicas numéricas tais como diferença e elementos finitos.

Para ilustrar a definição da transformada, considere por exemplo (caso bidimensional), $w(x, y)$ função em 2 variáveis com todas as derivadas parciais definidas e contínuas na origem. Definimos $\mathcal{D} w(x, y)=W(k, h)=\frac{1}{k ! h !} \frac{\partial^{k+h} f}{\partial x^{k} \partial y^{h}}(0,0)$ com $h$ e $k$ inteiros maiores ou iguais a zero. O domínio da transformada é, desta forma, o conjunto das funções de classe $\mathcal{C}^{\infty}\left(\mathbb{R}^{2}\right)$ e sua imagem o conjunto das funções definidas em $\mathbb{Z}^{+} \times \mathbb{Z}^{+}$.

Pelo Teorema de Taylor-Maclaurin para duas variáveis, é fácil ver que, nas condições anteriores, $w(x, y)=\sum_{k=0}^{\infty} \sum_{h=0}^{\infty} W(k, h) x^{k} y^{h}$. Considerando então a forma geral da EPD de $2^{\text {a }}$ ordem dada por $a(x, y) \frac{\partial^{2} u}{\partial x^{2}}+2 b(x, y) \frac{\partial^{2} u}{\partial x \partial y}+c(x, y) \frac{\partial^{2} u}{\partial y^{2}}+d(x, y) \frac{\partial u}{\partial x}+e(x, y) \frac{\partial u}{\partial y}+f(x, y) u=g(x, y)$, onde iremos supor que as funções $a(x, y), \cdots, g(x, y)$ são funções reais definidas em $\Omega \subseteq \mathbb{R}^{2}$ de classe $\mathcal{C}^{\infty}$ e assumimos, sem perda de generalidade $c(x, y) \neq 0$ (os casos onde $a(x, y) \neq 0$ ou $b(x, y) \neq 0$ quando $c(x, y)=0$ são tratados analogamente).

Denotaremos $A(k, h), B(k, h), \cdots, G(k, h)$, respectivamente, os termos das transformadas diferenciais das funções $a(x, y), b(x, y), \cdots, g(x, y)$ e, para fixar notação, assumiremos que as condições de contorno são dadas por $u(x, y)=p(x, y)$ e $\nabla u(x, y) \cdot \vec{n}=q(x, y)$ ao longo de $\partial \Omega$. Os termos das transformadas de $p(x, y)$ e de $q(x, y)$ são, respectivamente, denotadas por $P(k, h)$ e $Q(k, h)$ e, finalmente, os termos da transformada da função $u(x, y)$ são denotados por $U(k, h)$. Com essa notação, as etapas da solução numérica das EDPs de segunda ordem apresentadas são:

1. Calcule os termos $A(k, h), \cdots, G(k, h)$ das transformadas das funções $a(x, y), \cdots, g(x, y)$ e os 
termos $P(k, h), Q(k, h)$ das condições de contorno. Como as funções são todas conhecidas, esses termos serão um conjunto de valores numéricos.

2. Com os resultados dos Teoremas anteriores, escreva a relação de recorrência que calcula os termos $U(k, h)$.

3. Com uma quantidade suficiente de termos calculados, aproxime a numericamente a solução $u(x, y)$ da EDP por $u(x, y) \cong \sum_{i=0}^{k_{\max }} \sum_{j=0}^{j_{\max }} U(k, h) x^{i} y^{j}$

No caso aqui estudado, a ordem da computação desta recorrência é dada por:

$\begin{array}{lll}U(0,2), & U(1,2), U(2,2), & \ldots \\ U(0,3), & U(1,3), U(2,3), & \ldots \\ U(0,4), & U(1,4), U(2,4), & \cdots \\ \ldots & & \end{array}$

Para ilustrar o método, determinamos a distribuição de calor ao longo do tempo de uma placa retangular (ou seja, $T=T(x, y ; t)$ ) com comprimento $L=50(\mathrm{~cm})$ e largura $W=50(\mathrm{~cm})$. A barra encontra-se em regime estacionário $\left(\frac{\partial T}{\partial t}=0\right)$ três de seus lados são mantidos na temperatura $0^{\circ} \mathrm{C}(\mathrm{Cel}-$ sius) e o outro lado na temperatura $100^{\circ} \mathrm{C}$. A solução obtida pelo método das diferenças finitas aplicado à equação de Fourier (com parâmetros de condução iguais a unidade) correspondente utilizando uma malha de $100 \times 100$ elementos teve um erro (medido pela integral da diferença entre o valor numérico obtido e o valor da solução analítica) foi inferior a $5 \%$ em um tempo de CPU em torno de 30 segundos utilizando implementação em MATLAB. O mesmo erro (com malha idêntica) obtido pelo método dos elementos finitos - também em torno de 30 segundos - foi de $1,8 \%$.

Para aplicação da DT, kmax e hmax foram tomados, ambos, iguais a $10^{\text {a }}$ potência. A solução obtida em tempo inferior a 1 segundo teve, neste caso, erro de $0,03 \%$, ou seja, um erro 60 vezes menor obtido de forma 30 vezes mais rápida. Perturbações da solução pela introdução de ruído não modificaram de forma significativa tais resultados.

Como conclusão, este exemplo demonstra que a estabilidade e precisão das soluções obtidas utilizando DT é, como descrito na literatura [1,2], maior que as soluções equivalentes obtidas utilizando métodos padrões (diferenças e elementos finitos). Isto ocorre por que, neste último método, equações de recorrência algébricas definem aproximações sucessivas das soluções. Também de acordo com os resultados apresentados na literatura, a complexidade computacional tem sido menor com a aplicação da Transformada Diferencial alternativamente a Elementos Finitos ou Diferenças Finitas.

Ressaltamos como um fator de dificuldade no uso desta técnica e que as condições de fronteira complexas podem tornar a obtenção dos termos iniciais da recorrência inviáveis ou bastante complicados e que a aproximação com a transformada inversa envolvendo muitos termos (potências elevadas de $x^{k} y^{h}$ ) podem conduzir a um aumento tanto da imprecisão quando da dependência das condições iniciais.

Como perspectivas de trabalhos futuros, os autores pretendem aplicar a técnica na simulação numérica do comportamento de reatores perfeitamente agitados.

Palavras-chave: Equações Diferencias Parabólicas, Equação do Calor, Transformada Diferencial, Método das Diferenças Finitas, Método dos Elementos Finitos, Reatores Perfeitamente Agitados

\section{Referências}

[1] F. Ayaz, On the two-dimensional differential transform method, Appl. Math. Comput. 143(2-3) 361-374 (2003).

[2] F. Ayaz. Solution of the system of differential equations by differential transform method, Appl. Math. Comput. Vol. 147, pp. 547-67 (2004).

[3] N.T. Shawagfeh and D. Kaya, Comparing numerical methods for solutions of ordinary differential equations, Appl. Math. Lett., 17 323-328 (2004). 